\title{
Characterization of Optimal Plans for Stochastic Dynamic Programs*
}

\author{
LAWRENCE BLUME
}

Department of Economics, University of Michigan, Ann Arbor, Michigan 48109

David EASLEY

Department of Economics, Cornell University, Ithaca, New York 14853

AND

\section{MAUREen O'HARA}

Graduate School of Business and Public Administration, Cornell University. Ithaca. New York 14853

\begin{abstract}
This paper provides general techniques for the characterization of optimal plans resulting from stochastic dynamic programming. We show that under standard assumptions the optimal plans in both finite and infinite horizon problems can be obtained by an application of the Implicit Function Theorem to first order conditions. Further, we show that under certain checkable conditions, optimal plans and value functions are $p$-times differentiable for any integer $p \geqslant 0$. Finally, we apply our technique to obtain a $C^{p}$ plan and value function in a one sector infinite horizon growth problem under uncertainty. Journal of Economic Literature Classification Numbers: 022, 111, 213.
\end{abstract}

The analysis of many problems in economics requires the consideration of both time and uncertainty. One of the standard tools for solving such problems is stochastic dynamic programming. A frequent criticism of the application of this technique to economic decision problems is that although solutions are shown to exist they are not adequately characterized. The aim of this paper is to rebut this criticism for a broad class of finite and infinite horizon stochastic dynamic programming problems. We show that under

* The authors wish to thank Mukul Majumdar, Daniel Spulber, Henry Wan and an anonymous referee for helpful comments. This research was supported by National Science Foundation Grant Soc 79-07201 and the Center for Mathematical Studies in Economics and Management Science, Northwestern University. 
standard assumptions, optimal plans can be obtained by an application of the Implicit Function Theorem to a system of first order conditions. Hence, plans can be shown to be differentiable and comparative statics results can be obtained. Further we show that under certain checkable conditions, optimal plans and value functions are $p$ times differentiable. The assumptions that we use are, for the most part, similar to those commonly used in economics for classical comparative static analysis, i.e., differentiability and concavity of the objective function and the constraints. As we demonstrate in the final section, our approach can be applied to the one sector infinite horizon growth problem under uncertainty.

\section{Optimal Plans for Finite and Infinite Horizon Problems}

In this section we setup the discrete time dynamic programming problem under uncertainty. We also state, without proof, two theorems regarding existence and continuity of optimal value functions and plans. Our analysis will build from these results.

DEFINITION 1. A stationary Markov decision model is characterized by the tuple $(S,(\Omega, \phi), A, U, g, b)$, where

(i) $S$ is the state space for the system, $s \in S$.

(ii) $\Omega$ is the random events space, $\omega \in \Omega$. Let $\mathscr{B}$ be the sigma-algebra on $\Omega$ and let $\phi: \Omega \times \mathscr{B} \rightarrow[0,1]$ define the Markov transition probability on $(\Omega, \mathscr{B})$.

(iii) $A$ is the action space for the decision maker, $a \in A$.

(iv) The function $U: S \times A \times \Omega \rightarrow R_{+}$is the immediate reward, i.e., $U(s, a, \omega)$ is the reward to the decision maker of taking action $a$ when the state is $s$ and the random event $\omega$ occurs.

(v) The function $g: S \times A \times \Omega \rightarrow S$ is the transition equation, i.e., $g(s, a, \omega)$ is tomorrow's state if the current state is $s$, current action is $a$, and tomorrow's realized random event is $\omega$.

(vi) The function $b(s, a, \omega) \geqslant 0$ is the constraint equation.

The objective of the decision maker is to choose a sequence of actions which will maximize his expected discounted (discount rate $\beta$ ) total return subject to his constraints. Let $X=S \times \Omega$ denote the state of the system, where $x_{n}=\left(s_{n}, \omega_{n}\right)$ is an element of the state space at date $n$. We will now make precise the notion of an optimal plan.

DEFINITION 2. (i) A plan is a sequence $a=\left(a_{n}\right)$ of measurable maps $a_{n}: X \times \cdots_{n} \times X \rightarrow A$ with the property that $b\left(s_{n}, a_{n}\left(x_{1}, x_{2}, \ldots, x_{n}\right), \omega_{n}\right) \geqslant 0$ 
for $x_{n} \in X$, for all $n$. Let $\mathscr{A}$ denote the set of all plans. (ii) A Markov plan is a plan $a=\left(a_{n}\right)$ such that, for each $n, a_{n}\left(x_{1}, \ldots, x_{n}\right)$ depends only on $x_{n}$. (iii) A stationary plan, denoted $a=a^{(\infty)}$, is a Markov plan with the property that $a_{n}=a^{(\omega)}$ for all $n$.

DEFINITION 3. Given the plan a, an expected discounted total return for the $N$ period problem $(1 \leqslant N \leqslant \infty)$ is

$$
I^{N}(a)(x)=E\left[\sum_{n=1}^{N} \beta^{n-1} U\left(s_{n}, a_{n}\left(x_{1}, \ldots, x_{n}\right), \omega_{n}\right)\right], \text { where } x_{1}=x .
$$

Definition 4. The value function for the $N$ period problem $(1 \leqslant N \leqslant \infty)$ is

$$
V^{N}(x)=\sup _{a \in \mathscr{\infty}} I^{N}(a)(x)
$$

Thus, the value function represents the maximum feasible total return to the decision maker.

Definition 5. A plan $a^{*}$ is optimal for the $N$ period problem if $I^{N}\left(a^{*}\right)=V^{N}$.

Let $C^{p}(X ; Y)=\{f: X \rightarrow Y: f$ is continuous, bounded and has $p$ continuous derivatives $\}$. If $Y=R$ define $C^{p}(X ; R)=C^{p}(X)$. Where the argument is clear $C^{p}(X)$ is written $C^{p}$.

In order to guarantee even the existence of an optimal plan additional assumptions are necessary. Assumptions from the following list will also be used in the proofs of continuity and differentiability of optimal plans.

Assumption 1. The spaces $S, A$ and $\Omega$ are Borel subsets of $R^{k}, R^{l}$ and $R^{q}$, respectively.

Assumption 2. (i) $A$ is compact, $S$ and $A$ are convex.

(ii) $U$ is bounded above

Assumption 3. (i) $U \in C^{p}(S \times A \times \Omega)$ for $p \geqslant 0$.

(ii) $U(.,, \omega)$ is concave and nondecreasing on $S \times A$, for all $\omega \in \Omega$.

(iii) $U(s, ., \omega)$ is strictly concave on $A$, for all $(s, \omega) \in S \times \Omega$.

Assumption $4 . \quad 0 \leqslant \beta<1$. 
Assumption 5. (i) $g \in C^{0}(S \times A \times \Omega)$.

(ii) $g=\left(g_{1}, g_{2}, \ldots, g_{n}\right)$ where each cordinate function $g_{i}(., ., \omega)$ is concave on $S \times A$ and, for all $a \in A, g_{i}(\cdot, a, \omega)$ is nondecreasing on $S$, for all $\omega \in \Omega$.

(iii) $g(., ., \omega) \in C^{p}(S \times A)$, for all $\omega \in \Omega$, for $p \geqslant 0$.

Assumption 6. (i) $b \in C^{p}\left(S \times A \times \Omega ; R^{m}\right)$ for $p \geqslant 0$.

(ii) Let $\Gamma(x)=\{a \in A \mid b(s, a, \omega) \geqslant 0\}$. Then $\Gamma$ is a continuous correspondence, $\Gamma(x)$ is a convex set for all $x \in X$, and $\Gamma(s, w) \subset \Gamma\left(s^{\prime}, w\right)$ for $s^{\prime} \geqslant s$ and $\Gamma(\cdot, \omega)$ has a convex graph, for all $\omega \in \Omega$.

Assumption 7. (i) $\left\{\omega^{n}\right\}$ follows a stationary Markov process with transition probability $\phi: \Omega \times \mathscr{B} \rightarrow[0,1]$ and $\phi(\omega,$.$) is a weakly continuous$ function of $\omega$. $p \geqslant 0$.

(ii) For all $A \in \mathscr{B}, \phi(\omega, A)=\int_{A} f(\omega, \tilde{\omega}) d \tilde{\omega}$ and $f \in C^{p}(\Omega \times \Omega)$ for

Given Assumptions 1, 2, 3(i) and (ii), 4, 5, 6 and 7(i) it is known that a continuous value function and an optimal plan exist for any $N \leqslant \infty$. To state these results we first define an operator $T$ which generates a value function. Let $C_{s}^{p}(X)=\left\{f \in C^{p}(X): f\right.$ is concave and nondecreasing on $\left.S\right\}$. Define a norm on $C_{s}^{p}(X)$ by $\|f\|=\sup |f(x)|+\sup \|D F(x)\|+\cdots+\sup \left\|D^{p} f(x)\right\|$.

With this norm $C_{s}^{p}(X)$ is a Banach space. Define the operator $T$, for $V \in C_{s}^{p}(X)$, by

$$
T V(x)=\sup _{a \in A}\left\{U(s, a, \omega)+\beta \int V(g(s, a, \tilde{\omega}), \tilde{\omega}) d \phi(\omega, \tilde{\omega}) \mid 0 \leqslant b(s, a, \omega)\right\} .
$$

The following two Theorems present standard results about the operator $T$, the value function, and optimal plans. See Benveniste and Scheinkman [1], Blackwell [2] and Maitra [6].

THEOREM 1.1. Given assumptions 1, 2, 3(i) and (ii), 4, 5, 6 and 7(i) and $N=\infty$ :

(i) $T: C_{s}^{0}(X) \rightarrow C_{s}^{0}(X)$.

(ii) $T$ is a contraction map on $C_{s}^{0}(X)$ and $T$ has a unique fixed point $V^{\infty}$ which is the value function for the infinite horizon problem.

(iii) There exists an optimal plan for the infinite horizon problem, it is stationary and is given by solutions to:

$$
\max _{a \in A}\left\{U(s, a, \omega)+\beta \int V^{\infty}(g(s, a, \tilde{\omega}), \tilde{\omega}) d \phi(\omega, \tilde{\omega}) \mid b(s, a, \omega) \geqslant 0\right\} .
$$


(iv) If we add $\mathrm{A} 3(\mathrm{iii})$, there exists a unique optimal plan $a: X \rightarrow A$ for the infinite horizon problem and further $a \in C^{0}(X ; A)$.

The following theorem provides an initial characterization of the value function and optimal plan for the finite horizon problem. Let $T^{n} f=T\left(T^{n-1} f\right)$ for $n \geqslant 1$ and $T^{0} f \equiv 0$.

THEOREM 1.2. Given the assumptions of Theorem 1.1 and $1 \leqslant N<\infty$ :

(i) The value function for the $N$ period problem is given by

$$
V^{N}(x)=\max _{a \in A}\left\{U(s, a, \omega)+\beta\left[T^{N-1} O(g(s, a, \tilde{\omega}), \tilde{\omega}) d \phi(\omega, \tilde{\omega}) \mid b(s, a, \omega) \geqslant 0\right\}\right.
$$

where $O(g(s, a, \tilde{\omega}), \tilde{\omega}) \equiv 0$.

(ii) There exists an optimal plan $a=\left(a_{n}\right)$ for the $N$ period problem, it is Markov and is given by solutions to

$$
\begin{aligned}
\max & \left\{U\left(s_{n}, a_{n}, \omega_{n}\right)+\beta^{n} \int V^{N-n}\left(g\left(s_{n}, a_{n}, \tilde{\omega}_{n+1}\right), \tilde{\omega}_{n+1}\right)\right. \\
& \left.\times d \phi\left(\omega_{n}, \tilde{\omega}_{n+1}\right) \mid b\left(s_{n}, a_{n}, \omega_{n}\right) \geqslant 0\right\}
\end{aligned}
$$

where $V^{0}(\cdot) \equiv 0$ and $s_{n+1}=g\left(s_{n}, a_{n}, \tilde{\omega}_{n+1}\right)$ for $n=1, \ldots, N$.

(iii) If we add Assumption 3(iii) then there exists a unique optimal plan for the $N$ period problem,

$$
a=\left(a_{1}, \ldots, a_{N}\right) \quad \text { and further } \quad a_{n} \in C^{0}(X ; A) \quad \text { for all } n .
$$

Although the theorems presented in this section demonstrate the existence and continuity of value functions and optimal plans for both the finite and infinite horizon problems, they provide no apparent means for characterizing either value functions or optimal plans. We know from Theorems 1.1(iii) and 1.2(ii) that the optimal plans must solve a stated maximization problem, but this problem involves a value function about which very little is known. At this stage the value function has not been shown to be differentiable, so classical maximization techniques cannot be applied to find optimal plans. In the next two sections we will show, for a wide class of problems, that the value function and optimal plans are $C^{p}$, that optimal plans can be obtained by classical techniques and that these plans can be characterized by an application of the Implicit Function Theorem. 


\section{Characterization of Finite Horizon Optimal Plans}

The analysis of the previous section presupposed only continuity and concavity properties of the various functions involved in defining a dynamic programming problem. In this section we explore the implications of $C^{p}$ assumptions for $p \geqslant 1$. We will characterize the differentiability properties of the functions in the range of the operator $T$. These characterizations can then be used to derive first order necessary and sufficient conditions for optimal plans. Although similar first order conditions have been shown to characterize optimal plans in a number of specific problems (primarily in the optimal growth literature; see $[3,4,8,9])$ the results stated here are general and can be applied to many problems. Further, we obtain $C^{p}$ results for both optimal plans and value functions.

To simplify the analysis we assume that optimal actions satisfy the constraint $b$ with equality so that $b$ can be treated as an equality constraint. For most economic problems, including the example in Section 4, this involves no loss of generality. We first show that when interior solutions result, the value function is a $C^{1}$ function on $S$ if $p \geqslant 1$. For this result we need the following assumption.

Assumption 8. $g(s, a, \tilde{\omega})=\hat{g}(h(s, a), \tilde{\omega})$, where $h \in C^{p}\left(S \times A ; R^{n}\right)$ and $\hat{g} \in C^{p}\left(R^{n} \times \Omega ; S\right)$.

Define $G: S \times A \times \Omega \rightarrow R^{m+n}$ by

$$
G(s, a, \omega)=\left[\begin{array}{l}
b(s, a, \omega) \\
h(s, a) .
\end{array}\right.
$$

Then for all $(s, a, \omega) \in S \times \operatorname{Int} A \times \Omega, D_{a} G(s, a, \omega)$ is surjective.

Note that this assumption implicitly requires $l \geqslant m+n$ and is a checkable condition for any specified $b$ and $g$.

Choose $V \in C_{s}^{0}(X)$ and let $X_{\text {int }}=\{x \in \operatorname{Int} S \times \Omega: a(x) \in \operatorname{Int} A\}$, where $a: X \rightarrow A$ is the solution to

$$
\sup _{a \in A}\left\{U(s, a, \omega)+\beta \int V(g(s, a, \tilde{\omega}), \tilde{\omega}) d \phi(\omega, \tilde{\omega}): b(s, a, \omega)=0\right\} .
$$

TheOREM 2.1. Given assumptions 1 to $6,7(\mathrm{i})$, and 8 and $p \geqslant 1, D_{s} T V$ exists and is continuous on $X_{\mathrm{int}}$.

This theorem follows directly from a lemma due to Benveniste and Scheinkman [1]. For a similar result in the context of a one sector model see Harris [5]. The proof of Theorem 2.1 is given in the Appendix.

Theorem 2.1 has the following important corollary. Let 
$X_{n}^{N}=\left\{x \in \operatorname{Int} S \times \Omega: a_{n}(x) \in \operatorname{Int} A\right.$, where $a_{n}(x)$ is the $n$th period optimal plan for the $N$ period problem $\}$. Let $\hat{X}^{N}=\left\{x \in X_{1}^{N}\right.$ : $\left(g\left(s_{n}, a_{n}\left(x_{n}\right), \tilde{\omega}_{n+1}\right), \tilde{\omega}_{n+1}\right) \in X_{n+1}^{N}$ for almost every $\tilde{\omega}_{n+1} \in \Omega, \phi\left(\omega_{n}, \cdot\right)$, for all $n=1, \ldots, N-1\}$.

COROLlary 2.1. Given the assumptions of Theorem 2.1. For any given $N, 1 \leqslant N \leqslant \infty$, if $x_{1} \in \hat{X}^{N}$, then a necessary condition that $a_{n}(x)=a^{\prime}$, for any $1 \leqslant n \leqslant N$, is that there exist $\lambda_{n} \in R^{m}$ such that

(i) $D_{a} U\left(s_{n}, a^{\prime}, \omega_{n}\right)+\beta \int D_{s} V^{N-n}\left(g\left(s_{n}, a^{\prime}, \tilde{\omega}_{n+1}\right), \tilde{\omega}_{n+1}\right)$

$D_{a} g\left(s_{n}, a^{\prime}, \tilde{\omega}_{n+1}\right) d \phi\left(\omega_{n}, \tilde{\omega}_{n+1}\right)-\lambda_{n} D_{a} b\left(s_{n}, a^{\prime}, \omega_{n}\right)=0$,

(ii) $b\left(s_{n}, a^{\prime}, \omega_{n}\right)=0$.

Proof. Since $x_{1} \in \hat{X}^{N}, a^{n}\left(x^{n}\right) \in \operatorname{Int} A$ for all $1 \leqslant n \leqslant N$. For $N<\infty$, $V^{N-n}$ is given by Theorem 1.2 and hence it follows from Theorem 2.1 that $D_{s} V^{N-n}$ exists and is continuous. For $N=\infty$ it follows from Theorem 2.1 and the fact that $T V^{\infty}=V^{\infty}$ that $D_{\varepsilon} V^{\infty}$ exists and is continuous. Thus both the maximand and the constraints for the $N$ period problem are $C^{1}$ in $a$. Conditions (i) and (ii) are the classical necessary conditions of Lagrange for maximizing a $C^{1}$ function subject to non-degenerate $C^{1}$ equality constaints.

Q.E.D.

This corollary is useful because it is often possible to state a priori conditions guaranteeing that $\hat{X}^{N}$ is nonempty and contains most of the interesting points in $X$. The Inada conditions that are often used in optimal growth models yield a nontrivial $\hat{X}^{N}$, for an example see Majumdar and Zilcha $[8 \mid$.

Although Theorem 2.1 and its Corollary provide necessary conditions when optimal plans are interior, these equations have not previously been shown to be differentiable. Hence, they cannot easily be used to characterize optimal plans. In the remainder of this section we demonstrate that, for certain finite horizon problems, these equations are differentiable and optimal plans and value functions are $C^{p}$.

The next theorem makes use of the envelope theorem to study differentiability properties of functions in the range of $T$. Define a Lagrangian for the maximization problem, $L: R^{m} \times S \times A \times \Omega \rightarrow R$, by

$$
\begin{aligned}
L(\lambda, s, a, \omega)= & U(s, a, \omega)+\beta \int V(g(s, a, \tilde{\omega}), \tilde{\omega}) d \phi(\omega, \tilde{\omega}) \\
& +\lambda^{\prime} b(s, a, \omega) .
\end{aligned}
$$

For $V \in C_{s}^{p}(X), p \geqslant 0$, the optimization problem clearly has a solution $a(x)$. Hence, for $V \in C_{s}^{p}(X), x \in X_{\text {int }}, p \geqslant 1$ and $D_{a} b(s, a(x), \omega)$ of maximal rank the first order conditions $D_{(a, \lambda)} L(\lambda, s, a, \omega)=0$ have solutions $a(x)$ and $\lambda(x)$. 
Condition 1. Suppose that $V \in C_{s}^{p}(X), x \in X_{\mathrm{int}}, p \geqslant 2$, and along $a(x)$ and $\lambda(x), D_{a} b(s, a(x), \omega)$ is of maximal rank and $D_{(a, \lambda)}^{2} L(\lambda(x), s, a(x), \omega)$ is surjective.

This type of assumption is standard in classical optimization and is checkable for specific stochastic dynamic programming problems. In many problems the surjectivity condition can be verified directly from the properties of $U, g, b$ and the concavity of $V$, and hence it need not be checked recursively. The optimal growth example at the end of this paper is a problem satisfying this assumption.

THEOrem 2.2. Assume A1 to A7(ii) and Condition 1. Then $T: C_{s}^{p}(X) \rightarrow C^{p}\left(X_{\mathrm{int}}\right)$.

Proof. It follows from standard classical optimization that if $x^{\prime} \in X_{\text {int }}$ then a necessary condition for $a\left(x^{\prime}\right)=a^{\prime}$ is $D_{(a, \lambda)} L\left(\lambda^{\prime}, s^{\prime}, a^{\prime}, \omega^{\prime}\right)=0$ for some $\lambda^{\prime} \in R^{m}$. Since $U$ and $V$ are continuous in $a$ and $\Gamma(x)$ is compact there exist optimal plans, hence the first order conditions have solutions $a(x)=a^{\prime}$ and $\lambda(x)=\lambda^{\prime}$. By Condition $1, L$ is $C^{2}$ and $D_{(a, \lambda)}^{2} L\left(\lambda^{\prime}, s^{\prime}, a^{\prime}, \omega^{\prime}\right)$ is surjective, hence by the Implicit Function Theorem the optimal $a: X \rightarrow A$ and $\lambda: X \rightarrow R^{m}$ are locally $C^{p-1}$. In particular, since the optimal $a$ is unique and locally $C^{p-1}$ on $X_{\text {int }}, a \in C^{p-1}\left(X_{\text {int }}\right)$.

Returning to the Lagrangian, observe that, for each $(s, \omega) \in S \times \Omega$

$$
\begin{aligned}
T V(s, w) & =U(s, a(s, \omega), \omega)+\beta \int V(g(s, a(s, \omega), \tilde{\omega}), \tilde{\omega}) f(\omega, \tilde{\omega}) d \tilde{\omega} \\
& =L(\lambda(s, \omega), s, a(s, \omega), \omega) \quad \text { since } b(s, a(s, \omega), \omega)=0 .
\end{aligned}
$$

Hence,

$$
\begin{aligned}
D_{s} T V(s, \omega)= & D_{\lambda} L(\lambda(s, \omega), s, a(s, \omega), \omega) \cdot D_{s} \lambda(s, \omega) \\
& +D_{a} L(\lambda(s, \omega), s, a(s, \omega), \omega) \cdot D_{s} a(s, \omega) \\
& +D_{s} L(\lambda(s, \omega), s, a(s, \omega), \omega) .
\end{aligned}
$$

Since $D_{\lambda} L(\lambda(s, \omega), s, a(s, \omega), \omega)=0$ and $D_{a} L(\lambda(s, \omega) s, a(s, \omega), \omega)=0$ we have $D_{s} T V(s, \omega)=D_{s} L(\lambda(s, \omega), s, a(s, \omega), \omega)$. Since $L$ is $C^{p}, D_{s} L$ is $C^{p-1}$. Hence $D_{s} T V(s, \omega)$ is $C^{p-1}$. A similar envelope theorem argument shows that $D_{\omega} T V(s, \omega)$ is $C^{p-1}$. Hence we conclude that $T V$ is $C^{p}$ on $X_{\text {int }}$. Q.E.D.

Corollary 2.2. Assume A1 to A7(ii) and Condition 1 with $V$ replaced by the value function $V^{N-n}$. If $V^{N-n} \in C_{s}^{p}(X)$ then the optimal plan is $a_{n} \in C^{p-1}\left(X_{n}^{N}, A\right)$.

By a simple induction argument we also have: 
Corollary 2.3. Assume Al to A7(ii) and $p \geqslant 2$. For the $N$ period problem, $N<\infty$, let $L^{N-n}\left(\lambda_{n}, s_{n}, a_{n}, \omega_{n}\right)=U\left(s_{n}, a_{n}, \omega_{n}\right)+\beta \int V^{N-n}\left(g\left(s_{n}\right.\right.$, $\left.\left.a_{n}, \tilde{\omega}_{n+1}\right), \tilde{\omega}_{n+1}\right) \quad f\left(\omega_{n}, \tilde{\omega}_{n+1}\right) d \tilde{\omega}_{n+1}+\lambda_{n}^{\prime} b\left(s_{n}, a_{n}, \omega_{n}\right)$, for $n=1, \ldots, N$. Suppose that $x_{1} \in \hat{X}^{N}$ and that for each $n=1, \ldots, N$, along $a_{n}\left(x_{n}\right)$ and $\lambda_{n}\left(x_{n}\right), D_{a} b\left(s_{n}, a_{n}\left(x_{n}\right), \omega_{n}\right)$ is of maximal rank and $D_{\left(a_{n} \cdot \lambda_{n}\right)}^{2} L\left(\lambda_{n}\left(x_{n}\right)\right.$, $\left.s_{n}, a_{n}\left(x_{n}\right), \omega_{n}\right)$ is surjective. Then for all $n=1, \ldots ., N$ :

(i) $V^{N-n} \in C^{p}\left(X_{n}^{N}\right)$,

(ii) $a^{n} \in C^{p-1}\left(X_{n}^{N} ; A\right)$.

For problems with a finite horizon Theorem 2.2 and Corollaries 2.2 and 2.3 demonstrate that the value function and optimal plans are well behaved. The optimal plan can now be characterized by an application of the Implicit Function Theorem to the system of first order conditions defining the optimal action. More important for many applications is the result that if $x_{1} \in \hat{X}^{N}$ then optimal plans are $C^{p-1}$ and the value function is $C^{p}$, for any $p \geqslant 2$.

\section{Characterization of Infinite Horizon Optimal Plans}

For finite horizon problems the result of Theorem 2.2 that $T: C_{s}^{2}(X) \rightarrow C^{2}\left(X_{i n t}\right)$ is sufficient to demonstrate differentiability of optimal plans. For infinite horizon problems this result is not sufficient; essentially, we must show that $\lim _{n \rightarrow \infty} T^{n} V \in C^{2}\left(X_{\text {int }}\right)$, for any $V \in C_{s}^{2}(X)$. A direct method for doing so is to show that $T$ is a contraction map on $C^{2}(X)$. Yet, although intuitively appealing, it is difficult to establish reasonable sufficient conditions for this approach. Fortunately, however, there is another method of establishing the necessary properties of $T$. In this section, we use the uncertainty about future states given by the transition equation and the random events to "smooth out" possible discontinuities in the derivatives of $V$. Approaching the problem this way only requires assumptions that are analogous to the standard assumptions made on the stochastic processes used in continuous time optimization problems.

Assumption 10. (i) $\Omega=\Omega_{1} \times \Omega_{2}$ and we write $g(s, a, \omega)=g\left(s, a, \omega^{\prime}\right)$, $b(s, a, \omega)=b\left(s, a, \omega^{2}\right)$ and $U(s, a, \omega)=U\left(s, a, \omega^{2}\right)$ to indicate that only $\omega^{1}$ enters $g$ and only $\omega^{2}$ enters $b$ and $U$.

(ii) $\omega^{1}$ and $\omega^{2}$ are independent with densities $f_{1} \in C^{p}\left(\Omega_{1}\right)$ and $f_{2} \in C^{p}\left(\Omega_{2}\right), p \geqslant 1$.

(iii) $g: S \times A \times \Omega_{1} \rightarrow S$ is invertible on $\Omega_{1}$ with inverse $h \in C^{p}\left(S \times A \times S ; \Omega_{1}\right), p \geqslant 1$, where for any $(s, a) \in S \times A, h(s, a, \cdot)$ is a one-to-one map of $S$ into $R^{q}$. 
The structure of the uncertainty and the transition equation in Assumption 10 allows a wide range of problems. The crucial part of this assumption is that the random variable in the transition equation, $\omega^{1}$, has a $C^{p}$ density. Part (iii) is included only to insure that the random variable $s_{n+1}$ has a $C^{p-1}$ conditional density function. The global invertability of $g$ is convenient but certainly not necessary for this result.

The indirect transition law given by $g$ and $f_{1}$ implies a probability on $s_{n+1}$ given $s_{n}$ and $a_{n}$. Using Assumption 10 we can transform this indirect transition law into a direct transition law of the form $q(\cdot, s, a)$. If the original specification of the decision problem involves a transition law of this form, with a $C^{p-1}$ density, then Assumption 10 is unnecessary and Theorem 3.1 applies directly.

Let $B \in \mathscr{B} \mathscr{B}_{S}$ (the Borcl sets of $S \subset R^{k}$ ) and define

$$
\left.q(B \mid s, a) \equiv\right|_{h(s, a, B)} f_{1}\left(\tilde{\omega}^{1}\right) d \tilde{\omega}^{1}
$$

$q(B, s, a)$ is the probability that $s_{n+1} \in B$ given that $s_{n}=s$ and $a_{n}=a$.

Now $\int_{h(s, a, b)} f_{1}\left(\tilde{\omega}^{1}\right) d \tilde{\omega}^{1}=\int_{B} f_{1}(h(s, a, \tilde{s}))\left|D_{\tilde{s}} h(s, a, \tilde{s})\right| d \tilde{s}$. Define $r(s, a, \tilde{s}) \equiv f_{1}(h(s, a, \tilde{s}))\left|D_{\tilde{s}} h(s, a, \tilde{s})\right|$ and note that $r \in C^{p-1}(S \times A \times S)$. Now for any $B \in \mathscr{B}_{s}, q(B \mid s, a)=\int_{B} r(s, a, \tilde{s}) d \tilde{s}$.

We now want to exploit the properties of the value function derived earlier to write the problem of selecting an optimal action as a classical maximization problem. Note that this theorem requires only $x \in X_{\text {int }}$ (where $X_{\text {int }}$ is defined using $V^{\infty}$ ) and not $x \in \hat{X}^{\infty}$. The conditions used in this theorem are checkable for any specific problem, for example they are clearly satisfied in the infinite horizon optimal growth problem presented in the next section.

THEOREM 3.1. Given Assumption 1 to 6 and 10 and $S, \Omega$ compact; if $p>2$ then the Lagrangian, $L: R^{m} \times S \times A \times \Omega \rightarrow R$, defined by, $L(\lambda, s, a, \omega)=U\left(s, a, \omega^{2}\right)+\beta \int V^{\infty}\left(g\left(s, a, \tilde{\omega}^{1}\right), \tilde{\omega}^{2}\right)\left(f_{1}\left(\tilde{\omega}^{1}\right)\right.$, $\left.f_{2}\left(\tilde{\omega}^{2}\right)\right)\left(d \omega^{1}, d \omega^{2}\right)+\lambda^{\prime} b\left(s, a, \omega^{2}\right)$ is $C^{p-1}$ on $R^{m} \times S \times A \times \Omega$. Suppose that $D_{a} b\left(s, a(x), \omega^{2}\right)$ is of maximal rank for all $x \in X_{\text {int }}$. Then for any $x \in X_{\mathrm{int}}$, $D_{(a . .)} L\left(\lambda^{\prime}, s, a^{\prime}, \omega\right)=0$ is a necessary and sufficient condition for $a$ maximum at $\left(a^{\prime}, \lambda^{\prime}\right)$ in the infinite horizon problem and there exist solutions $a(x)=a^{\prime}$ and $\lambda(x)=\lambda^{\prime}$. If $D_{(a, \lambda)}^{2} L(\lambda, s, a, \omega)$ is surjective along $a(x)$ and $\lambda(x)$ for all $x \in X_{\mathrm{int}}$ then $V^{\infty} \in C^{p-2}\left(X_{\mathrm{int}}\right)$ and $a \in C^{p-2}\left(X_{\mathrm{int}}\right)$.

Proof. Define $M: S \times A \times \Omega \rightarrow R$ by $M(s, a, \omega)=U\left(s, a, \omega^{2}\right)+$

$$
\beta \int V^{\infty}\left(g\left(s, a, \tilde{\omega}^{1}\right), \tilde{\omega}^{2}\right)\left(f_{1}\left(\tilde{\omega}^{1}\right), f_{2}\left(\tilde{\omega}^{2}\right)\right)\left(d \tilde{\omega}^{1}, d \tilde{\omega}^{2}\right) .
$$


Using the results above it follows that $M(s, a, \omega)=U\left(s, a, \omega^{2}\right)+$ $\beta \int V^{\infty}\left(\tilde{s}, \tilde{\omega}^{2}\right)\left(r(s, a, \tilde{s}), f_{2}\left(\tilde{\omega}^{2}\right)\right)\left(d \tilde{s}, d \tilde{\omega}^{2}\right)$. Since $U \in C^{p}$ by assumption, $r \in C^{p-1}$ by the preceding argument and $V \in C^{0}$ by Theorem 1.1, we have $M \in C^{p-1}$. Now since $b \in C^{p}$ by assumption, $M \in C^{p-1}$ and $L(\lambda, s, a, \omega)-$ $M(s, a, \omega)+\lambda^{\prime} b(s, a, \omega)$ we have $L \in C^{p-1}$.

We can write the problem of finding an optimal plan for the infinite horizon problem as

$$
\operatorname{Max}_{a \in A}\left\{M(s, a, \omega): b\left(s, a, \omega^{2}\right)=0\right\}
$$

Since $U$ is concave on $S \times A, V^{\infty}$ is concave and nondecreasing on $S$ and $g$ is concave on $S \times A, M$ is concave on $S \times A$. For any $x \in X_{\text {int }}$ the constraint set, $\Gamma(x)$, is convex and $D_{a} b\left(s, a(x), \omega^{2}\right)$ is of maximal rank so a necessary and sufficient condition for an interior maximum at $\left(a^{\prime}, \lambda^{\prime}\right)$ is that $D_{(a, \lambda)} L\left(\lambda^{\prime}, s, a^{\prime}, \omega\right)=0$. By Theorem 1.1 there exists an optimal action $a^{\prime}$, hence the equation above has solutions $a(x)=a^{\prime}$ and $\lambda(x)=\lambda^{\prime}$. By the argument of Theorem 2.2 we also know that the optimal action $a$ and multiplier $\lambda$ are $C^{p-2}$ on $X_{\text {int }}$, if $D_{(a, \lambda)}^{2} L(\lambda, s, a, \omega)$ is surjective along $a(x)$, $\lambda(x)$, for all $x \in X_{\text {int }}$.

Now by definition $V^{\infty}(s, \omega)=L(\lambda(s, \omega), \quad s, \quad a(s, \omega), \omega)$ since $b\left(s, a(s, \omega), \omega^{2}\right)=0$. Since $L$ is $C^{p-1}$ and $a$ and $\lambda$ are $C^{p-2}$ on $X_{\text {int }}$ we have $V^{\propto} \in C^{p-2}\left(X_{\mathrm{int}}\right)$.

Q.E.D.

We have shown that optimal plans as well as comparative static results can be obtained by a direct application of the Implicit Function Theorem to the equation system

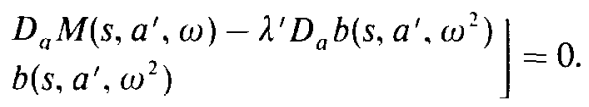

Further, optimal actions and the value function are $C^{p-2}\left(X_{\mathrm{int}}\right)$. The usefulness of this approach depends crucially on how "large" $X_{\text {int }}$ is in any particular problem. The example in the next section shows that at least in a one sector optimal growth problem with uncertainty, $X_{\text {int }}$ is very large indeed. A further example can be found in $\mathrm{O}^{\prime} \mathrm{Hara}|10|$, where this technique is applied to analyze decision making in the financial firm.

\section{An Example}

Consider a one sector growth model with stochastic production possibilities. The specific model that we use is adopted from Majumdar and 
Zilcha [8] which builds on the standard optimal growth literature. In the optimal growth problem the objective of the decision maker is to select consumption and investment policies to maximize expected discounted utility of consumption subject to stochastic production possibilities.

The state space for the system is represented as $S=[0, \infty]$, where $s_{n} \in S$ is the total output available to either consume or invest in period $n$ (we assume $s_{1}>$ ). The random events space is the closed interval of real numbers $\Omega=[\alpha, \gamma]$ for $0<\alpha<\gamma<\infty$. We assume that $\tilde{\omega}$ is distributed according to a density $h \in C^{p}([\alpha, \gamma])$. If in period $n$ an investment of $i \in[0, \infty]$ is made the output in period $n+1$ is stochastic and is given by the (net of depreciation) production function $g(i, \omega)$ mapping $S \times \Omega$ to $S$ such that:

Assumption 1. (i) For each $\tilde{\omega}, g(\cdot, \tilde{\omega})$ is $C^{p}((0, \infty))$ for $p>2$, $\infty>g_{i}(\cdot, \tilde{\omega})>0$ and

$$
g_{i i}(\cdot, \tilde{\omega})<0
$$

(ii) $g(i, \tilde{\omega})>0$ for all $\tilde{\omega}$, if $i>0$, and $g(0, \tilde{\omega})=0$ for all $\tilde{\omega}$.

(iii) $g(i, \cdot)$ is strictly increasing for all $i$.

(iv) $g_{i}(i, \tilde{\omega}) \rightarrow \infty$ as $i \rightarrow 0$, for all $\tilde{\omega}$. $g_{i}(i, \tilde{\omega}) \rightarrow 0$ as $i \rightarrow \infty$, for all $\tilde{\omega}$.

There exists an $\hat{\imath}>0$ such that $g(i, \tilde{\omega})<\hat{\imath}$ for all $i>\hat{\imath}$, for all $\tilde{\omega}$.

Formally, the decision makers objective is to select a sequence of actions $\left\{a_{n}\right\}=\left\{\left(c_{n}, i_{n}\right)\right\}$ to maximize $E \sum_{n=1}^{\infty} \beta^{n-1} U\left(c_{n}\right)$, where $0<\beta<1$ and

Assumption II. (i) $U \in C^{p}\left(R_{+}\right)$for $p>2, U^{\prime}(c)>0$, and $U^{\prime \prime}(c)<0$ for all $c$.

(ii) $U^{\prime}(c) \rightarrow \infty$ as $c \rightarrow 0$,

$U^{\prime}(c)<\infty$ for all $c>0$,

$U$ is bounded above.

The constraint that the planner faces in any period $n$ is $s_{n}-c_{n}-i_{n} \geqslant 0$. In this framework the stochastic production function $g(i, \omega)$ is the transition equation and the constraint equation is $b(s, c, i)=s-c-i=0$. (Since $U^{\prime}(c)>0$ the constraint $s-c-i \geqslant 0$ can be treated as $s-c-i=0$.)

It can be shown (see [8]) that under these assumptions there exist optimal actions $c(s, \omega)$ and $i(s, \omega)$ and further there exist $0<i<\infty$ and $0<\bar{c}<\infty$ such that $0 \leqslant c(s, w) \leqslant \bar{c}$ and $0 \leqslant i(s, w) \leqslant i$. Using this result we can represent the action space as the compact set $A=|0, \bar{c}+\varepsilon| \times|0, \bar{i}+\varepsilon|$, for some $\varepsilon, \infty>\varepsilon>0$. It is also easy to show, using Assumptions I and II, that 
if $s>0$ then $c(s, w)>0$ and $i(s, w)>0$ for all $w$. Hence, $X_{\text {int }} \subset\left(0, i^{*}\right) \times(\alpha, \gamma)$, for $i^{*}=\max g(i, \omega)$ over $0 \leqslant i \leqslant \bar{l}, \alpha \leqslant \omega \leqslant \gamma$.

Now, if $p>2$ and $s_{1} \varepsilon\left(0, i^{*}\right)$, the result of Theorem 3.1 clearly applies to this problem when A10 is met for a compact $X \subset\left[0, i^{*}\right] \times[\alpha, \gamma]$. Hence, the value function $V^{\infty}$ is $C^{p-2}\left(X_{\text {int }}\right)$ and optimal plans are characterized by the first order conditions,

$$
\begin{aligned}
& s-c^{\prime}-i^{\prime}=0, \\
& U^{\prime}\left(c^{\prime}\right)-\lambda^{\prime}=0, \\
& \beta E\left[V_{s}^{\infty}\left(g\left(i^{\prime}, \tilde{\omega}\right), \tilde{\omega}\right) \cdot g_{i}\left(i^{\prime}, \tilde{\omega}\right)\right]-\lambda^{\prime}=0 .
\end{aligned}
$$

Further the Jacobian of the first order conditions can easily be shown to be strictly positive, so $c(s, \omega)=c^{\prime}$ and $i(s, \omega)=i^{\prime}$ are $C^{p-2}$ on $X_{\text {int }}$.

The first order conditions that characterize optimal policies are well known for this optimal growth problem. However, the result that policies and the value function are $C^{p-2}$, for any $p>2$, is new and may be of independent interest. As this example illustrates, differentiable first order conditions and differentiability of policies and value functions are immediate results of our technique. Our technique, however, has applications far beyond the simple one sector optimal growth problem. It can be readily applied to a variety of intertemporal decision problems, including, for example, consumption and portfolio chocie problems and multisector optimal growth problems. To use this technique for such problems it is necessary to insure that optimal actions are interior. In the multisector growth problem interior actions will result if we make the obvious changes in Assumptions I and II and add an indecomposability assumption, see Majumdar and Radner $|7|$.

\section{APPENDIX}

The proof of Theorem 2.1 involves the following lemma; see Benveniste and Scheinkman $[1 \mid$.

Lemma 2.1. Let $U \subseteq R^{n}$ be an open convex set. Let $h: U \rightarrow R$ be a concave function. Suppose there exists $k: U \rightarrow R$ such that:

(i) $k$ is concave and continuously differentiable.

(ii) $k\left(u_{0}\right)=h\left(u_{0}\right)$ for some $u_{0} \in U$.

(iii) $k(u) \leqslant h(u)$ for all $u \in U$.

Then there exists a neighborhood $N$ of $u_{0}$ such that $h$ is continuously differentiable on $N$ and $D_{u} h\left(u_{0}\right)=D_{u} k\left(u_{0}\right)$.

Proof of Theorem 2.1. We simply construct a function $k$ satisfying 
(i)-(iii) of Lemma 2.1 with respect to $T V$, for fixed $\omega^{\prime} \in \Omega$. Choose $\left(s^{\prime}, \omega^{\prime}\right)=x^{\prime} \varepsilon X_{\text {int }}$, and let $a^{\prime}=a\left(x^{\prime}\right)$. Consider the system of equations

$$
\left.\begin{array}{l}
b\left(s, a, \omega^{\prime}\right) \\
h(s, a)-h\left(s^{\prime}, a^{\prime}\right)
\end{array}\right\rfloor=0 .
$$

By assumption the inverse function theorem is satisfied, so in an open neighborhood $N$ containing $s^{\prime}$ we can define $\hat{a}\left(\cdot, \omega^{\prime}\right) \in C^{1}(N ; A)$ such that

$$
\begin{aligned}
& \hat{a}\left(x^{\prime}\right)=a^{\prime} \\
& \left.\begin{array}{l}
b\left(s, \hat{a}\left(s, \omega^{\prime}\right), \omega^{\prime}\right) \\
h\left(s, \hat{a}\left(s, \omega^{\prime}\right)\right)-h\left(s^{\prime}, a^{\prime}\right)
\end{array}\right]=0 .
\end{aligned}
$$

Now we define $k: N \rightarrow R$ by

$$
k(s)=U\left(s, \hat{a}\left(s, \omega^{\prime}\right), \omega^{\prime}\right)+\beta \int V\left(g\left(s, \hat{a}\left(s, \omega^{\prime}\right), \tilde{\omega}\right), \tilde{\omega}\right) d \phi(\omega, \tilde{\omega}) .
$$

Observe that $\hat{a}$ is feasible for all $s \in N$. Hence $k(s) \leqslant T V\left(s, \omega^{\prime}\right)$. Next. observe that $\hat{a}\left(x^{\prime}\right)=a^{\prime}$ so that $k\left(s^{\prime}\right)=T V\left(x^{\prime}\right)$. Finally observe that $g\left(s, \hat{a}\left(s, \omega^{\prime}\right), \tilde{\omega}\right)=\hat{g}\left(h\left(s, \hat{a}\left(s, \omega^{\prime}\right)\right), \tilde{\omega}\right)=\hat{g}\left(h\left(s^{\prime}, a^{\prime}\right), \tilde{\omega}\right)$, so that for each $\tilde{\omega} \in \Omega, V\left(g\left(s, \hat{a}\left(s, \omega^{\prime}\right), \tilde{\omega}\right), \tilde{\omega}\right)$ is constant on $N$. Hence, since $U$ and $\hat{a}$ are $C^{\prime}$ functions on $N, k \in C^{1}(N)$. The function $k$ satisfies the assumptions of Lemma 2.1 with respect to $T V\left(\cdot, \omega^{\prime}\right)$, and so the theorem follows.

\section{REFERENCES}

1. L. M. Benveniste and J. A. Scheinkman, On the differentiability of value functions in dynamic models of economics, Econometrica 47 (1979).

2. D. Blackwell, Discounted dynamic programming, Ann. Math. Statist. 36 (1965).

3. W. A. BRock AND L. J. Murman, Optimal economic growth under uncertainty: The discounted case, J. Econ. Theory 4 (1972).

4. D. Easley and D. Spulber, Stochastic equilibrium and optimality with rolling plans, Internat. Econ. Rev. 22 (1981).

5. M. HARRIS, Notes for a Course in Dynamic Competitive Analysis, unpublished manuscript, Carnegie-Mellon University, Spring, 1980.

6. A. MaItra, Discounted dynamic programming on compact metric spaces, Sankhya, Ser. A 30, Part 2 (1968).

7. M. Majumdar and R. Radner, Stationary Optimal Policies with Discounting in a Stochastic Activity Analysis Model, Working Paper No. 186, Cornell University, 1979.

8. M. Majumdar AND I. Zilcha, On Optimal Accumulation Under Uncertainty in the Aggregative Framework: Some General Results, Manuscript, Cornell University, 1980.

9. T. Mitra, On optimal economic growth with variable discount rates: Existence and stability results, Internat. Econ. Rev. 20 (1979).

10. M. O'Hara, The Effect of Organization Structure on Economic Behavior: A Study of the Savings and Loan Industry, Ph.D Dissertation, Northwestern University, 1979. 